\title{
SIMULATION BASED PERFORMANCE TEST OF INCIDENT DETECTION ALGORITHMS USING BLUETOOTH MEASUREMENTS
}

\author{
Maria Karatsoli, Martin Margreiter, Matthias Spangler \\ Technical University of Munich \\ Munich, Germany, Arcistrasse 21 \\ 0030 6979838126, maria.karatsoli@gmail.com
}

\begin{abstract}
This article analyzes the use of Bluetooth-based travel times, for Automatic Incident Detection (AID) purposes. Automatic incident messages were derived for simulated data through the use of an AID algorithm, which was developed by Technical University of Munich (TUM). A Vissim model of a 15 kilometre section of A9 motorway in Germany was set up, where different scenarios of traffic situation, incidents and detector layout were introduced and travel times were generated, processed and then run through the TUM algorithm. The performance measures Detection Rate (DR), False Alarm Rate (FAR) and Mean Time To Detect (MTTD) were used for the analysis of the incident messages' quality of the simulated data and compared for every incident scenario. Local data were also generated in the Vissim model and used by $\mathrm{VK}_{\text {Diff }}$ algorithm for incident detection. A comparison of the quality of the incident messages of both TUM and $\mathrm{VK}_{\text {Diff }}$ algorithm was conducted.
\end{abstract}

Keywords: Simulation, Traffic incident, Automatic Incident Detection, Bluetooth, Incident scenarios, Detector layout

\section{Introduction}

Over the last years, the continuous increase in traffic demand brought the need for a more efficient traffic management system and especially for a more efficient traffic incident management system. More specifically, incident management systems aim at quick detection and removal of incidents in order to reduce their adverse effects. The Automatic Incident Detection (AID) approach is a fast incident detection method, which has as an input traffic data derived from detector technologies. The process of the input data is done through an AID algorithm which gives information about an incident existence. The variety of the available detector and algorithm technologies leads to a significant number of solutions for incident detection.

There are numerous algorithms that can be used for AID purposes, which need different types of data. The algorithm should be chosen carefully because its performance affects directly the performance of the whole incident management system. At this point, it is important to be mentioned that the main problem of AID algorithms is that they don't detect incidents but congestion resulting from them. As a result only incidents that cause congestion can be detected. The congestion, caused by the incidents is affected by factors concerning the incident (type, characteristics, severity), the environment (weather condition, visibility) and the location (type of the road, availability of lanes). Severe incidents that lead to insufficient capacity for the oncoming traffic can be easier detected than less severe incidents.

One of the promising detector technologies is the vehicle re-identification via Bluetooth, due to the increasing number of Bluetooth-enabled devices among the road users, their low maintenance and installation cost and the anonymity of detections. The system of Bluetooth detector technology is based on the concept of re-identifying vehicles at two measuring points and calculation of vehicle's travel time between them. When AID algorithms identify significant variations in these travel times due to an incident occurrence, they trigger an incident alarm. The performance of the AID algorithms and the quality of the incident messages is determined in terms of statistical performance measures false alarm rate (FAR), detection rate (DR) and mean time to detect (MTTD).

\subsection{Performance Indicators of AID Algorithms}

The following indicators are mostly used for the evaluation of the incident detection:

- Detection Rate (DR): is defined as the percentage of the number of detected incident cases to the total number of actual incident cases and describes the successfulness of an algorithm. 


$$
D R=\frac{\text { No. of detected incident cases }}{\text { Total No. of actual incident cases }} * 100 \%
$$

- False Alarm Rate (FAR): has different definitions for different applications. The most common one is the percentage of the number of false alarms to the total number of alarms, computed as:

$$
F A R=\frac{\text { No. of false alarms }}{\text { Total No. of alarms }} * 100 \%
$$

- Mean Time to Detect (MTTD): is the average time required for the algorithm to detect an incident. It is often expressed in units of minutes. Formula of MTTD is given in the following equation:

$$
M T T D=\frac{\sum_{i=1}^{N_{\text {det }}}\left(\mathrm{t}_{\mathrm{d}}-\mathrm{t}_{\mathrm{inc}}\right)}{\mathrm{N}_{\mathrm{det}}} * 100 \%
$$

where, $\mathrm{N}_{\text {det }}$ is the number of detected incidents, $t_{d}$ is the time that an incident is detected and $t_{\text {inc }}$ is the time that an incident is occurred.

In general, DR and FAR reflect the effectiveness of AID, while MTTD reflects the efficiency. The DR and MTTD are considered generally the most important evaluation factors of an incident detection system. Nevertheless, it is important the consideration of FAR in order to assure the reliability of the system (Shiereck, 2006). Low FAR is needed because warnings without an actual accident can make drivers ignore them in the future. Performance measures DR, FAR and MTTD are not independent. The desirable low FAR can be achieved through lower DR which is opposing to the ultimate goal of the highest possible values for DR.

\subsection{TUM Algorithm}

For AID purposes, the Technical University of Munich (TUM) developed an algorithm which uses travel time measurements (Margreiter, 2010; Margreiter et al., 2015). This TUM algorithm belongs to the category of spatial measurement-based algorithms and detects changes in travel times that could lead to an incident occurrence. Possible disturbances in traffic flow are expressed through increasing travel times that lead to significant drop of desirable speeds. For the determination of travel times between two detection sections, data from every single vehicle are used. More specifically, the exact time of every vehicle at each detector section, the number of the respective detector as well as the ID of the vehicle are taken into consideration.

\section{Study Area and Database}

Field data from the so called iRoute project were used for the design of the Vissim model. The scope of that project was to examine and compare different detector systems, by collecting data for real time traffic, in order to provide the best service of traffic information and to assure the optimal use of the Variable Message Signs (Listl and Singer, 2012). For that reason different traffic detectors were installed on a 15 kilometre section on the A9 (Autobahn 9) motorway near Nuremberg, Germany. As the featured incidents and the layout of Bluetooth detectors don't cover a wide range, a Vissim model was set up for different detectors' layout and traffic conditions, to produce various scenarios.

\subsection{Study Area}

The road network used in this study is the above mentioned 15 kilometre road section of the A9 motorway, in Germany. This motorway extends from Berlin's Ring road A10 $(0+000 \mathrm{~km})$, passes through Dessau, Leipzig, Hermsdorfer Kreuz (where A9 is connected to A4), Hof, Nuremberg and ends up in Munich $(529+800 \mathrm{~km})$. 
The examined part is located close to Nuremberg, more specifically from $371+000 \mathrm{~km}$ to $386+000$ $\mathrm{km}$. Along this $15 \mathrm{~km}$ motorway section, four interchanges are included. At $372+800 \mathrm{~km}$ there is the main interchange-Nuremberg, where the A9 is connected to A3 motorway and at $380+900 \mathrm{~km}$ another main interchange, Nurnberg-East, where the A9 is connected to the A6. The other two interchanges are smaller and include the Nuremberg-Fischbach junction, which is located at $379+700 \mathrm{~km}$ and Nuremberg-Feucht at the end of the examined part.

\subsection{Database for the Design of the iRoute Testbed Vissim Model}

Vissim, traffic micro simulation software developed by PTV was used to simulate the traffic conditions of the examined study area. It was used for simulating and analyzing future potential traffic conditions in the examined network, including an incident occurrence. For the design of a realistic Vissim model a set of traffic and network related data is necessary. The traffic volumes per hour and the vehicle composition information, derived from laser detectors (on 18.04.2011) that were installed at five sites along the examined road section, in the context of the iRoute project.

After the complete design of the road sections, counters of vehicle travel times were placed to the exact positions of the Bluetooth detectors. Then the available vehicle volumes and traffic composition were introduced at the beginning of each road link. Also, the desired speed decisions were placed at the appropriate points on the network and equipped with the respective speed distributions from travel speed data based on the Bluetooth detectors that were placed at three different points during the iRoute project.

A total number of 10 simulation runs is decided, since the results of a single simulation run are considered unreliable for further analysis. Also, traffic data would start to be registered by Vissim after 1800 simulation seconds. Since Vissim simulation starts with an empty network a warm-up period is required so that the network is completely full with vehicles. After running 10 simulations a set of travel time results between the Bluetooth detectors in the modelled traffic network was generated.

\subsection{Calibration of the Vissim Model}

The model was calibrated to ensure a validated representation of reality and traffic patterns and hence realistic results. The micro simulation model contains many adjustable parameters that are used as calibration parameters, such as speed distribution and car-following model parameters.

The average travel time per hour, measured by the Bluetooth detectors on 18.04 .2011 are the reference and basis for the calibration process. The calibration parameters listed above were altered through an iterative process until the travel time in 504-506 and 504-510 reaches a value within 15\% (Wisconsin Department of Transportation, 2002) of the measured travel time considered as our reference value.

On 18.04.2011 an incident of 27 minutes occurred from 8:39 to 9:06. In general, a calibration of an incident is difficult to be achieved on micro simulation models and requires accurate and detailed data. The following Figures show the speed diagrams from the actual (at the left) and simulated (at the right) data for 504-506 and 504-510 sections, coming out from the TUM algorithm. An exact modelling of the incident was not achieved, due to lack of important and detailed data about the incident such as the queue length [m].More specifically, the incident based on the actual data was not detected by TUM algorithm at the section 504-510, while for the simulated data there is an incident detection. The inexact calibration of the incident is the main reason for that, in combination with the fact that most of the calibrated travel times for the section 504-510 were higher than the reference values. The lack of the exact traffic volumes at the exit ramps on this specific day, had also negative effect on the incident calibration.
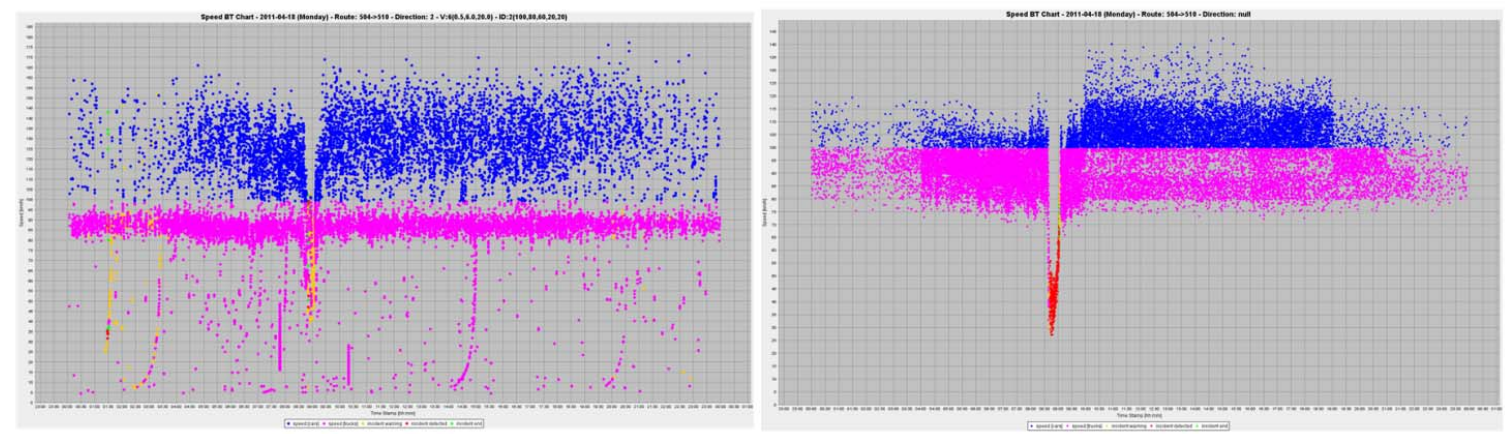

Figure 1. Speed graphs of section 504-506 on 18.04.2011, based on actual (left) and simulated data after calibration (right) 

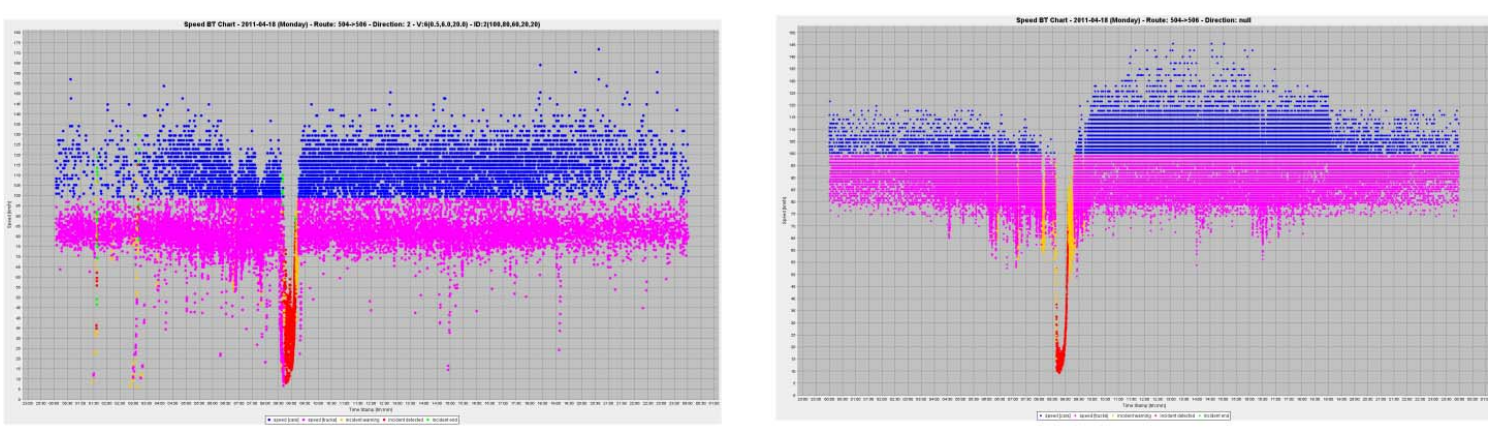

Figure 2. Speed graphs of section 504-510 on 18.04.2011, based on actual (left) and simulated data after calibration (right)

\section{Overview of Simulated Data}

Due to the limited availability of real data, the use of simulated data is necessary for the analysis of specific or future situations and incidents. In this case travel time data were obtained from five Bluetooth detectors. More specifically the sections examined by the TUM algorithm were from id504 $(377+839 \mathrm{~km})$ to id506 $(379+900 \mathrm{~km})$,id504 to id507 $(381+084 \mathrm{~km})$, id504 to id508 $(382+900 \mathrm{~km})$ and id504 to id510 $(385+900 \mathrm{~km})$ sensor.

Those four sections of $2.061 \mathrm{~km}(504-506), 3.245 \mathrm{~km}(504-507), 5.061 \mathrm{~km} \mathrm{(504-508)} \mathrm{and}$ $8.061 \mathrm{~km}$ (504-510) were examined under low-normal-high traffic volumes and the proposed incident scenarios. For the simulation of a lane blockage on Vissim a traffic light with a single-control fixed time signal (red and green sequence) was used. This allows simulating exact incident duration. Aim of these actions-incident scenarios, was to cause a congestion in all three lanes. In this study, an incident occurrence was considered only when a congestion of the three lanes was observed during the Vissim run for more that 15 minutes.

In total nine incident scenarios- actions were setup in Vissim at different locations along the examined road network and at different time during the day for different duration. For the first two scenarios, a desired speed area of $5-10 \mathrm{~km} / \mathrm{h}$ was implemented on the exit ramps at Nuremberg-Fischbach (PWC Brunn-West) and Nuremberg-East junctions, respectively. Incidents or accidents happening on the exit ramps as well as high exiting volumes could lead in reality to such sharp speed drops at the exits. The aim was the occurrence of an incident on the main road section in Vissim due to this speed reduction. Bad weather conditions or other warnings could lead to significant speed reduction. For that reason, a desired speed decision of 40-60 km/h was implemented at critical parts on the main road, during the third incident scenario. An accident without injuries or a stopped vehicle could lead to one lane blocking. Hence, the next three scenarios-actions include the closure of the right lane. The last three incident scenarios represent a more severe accident that could cause a two-lane closure. The specific location of each actionincident at the analyzed road section is shown on the following layout, see Figure 3.

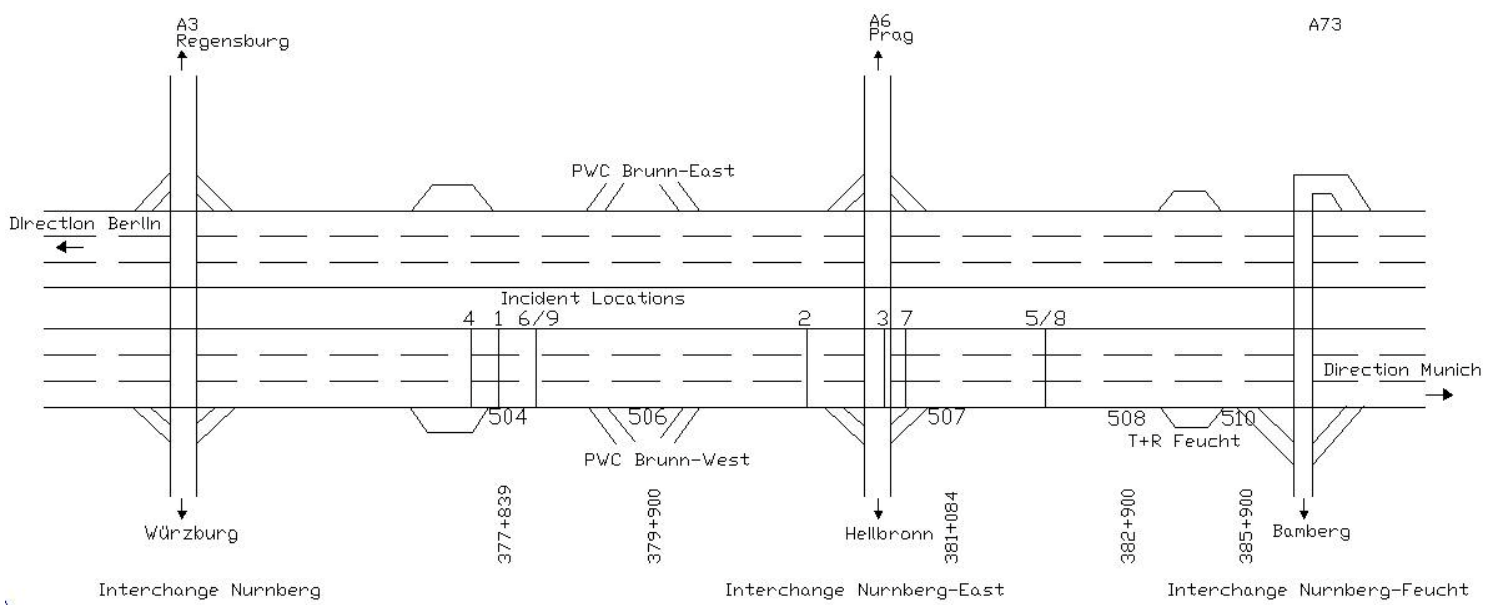

Figure 3. Location of incidents-actions on the Vissim model 
Not every incident scenario-action after the implementation in Vissim, under high-normal-low traffic volume, led to a significant reduction of capacity and congestion in the three lanes. More specifically, a desired speed area at the exit Nuremberg-Fischbach of $5-10 \mathrm{~km} / \mathrm{h}$ (scenario 1) affected the traffic flow on the main road only for high traffic volumes and not for normal and low. In contrast, a respective drop of speed at exit Nuremberg-East (scenario 2) affected the traffic flow in the three traffic situations, where an incident at $380+200 \mathrm{~km}$ on the main road has observed. The two scenarios $(5$ and 6$)$ of one lane closure during off-peak hours didn't affect the traffic flow under the three traffic situations, so an actual incident of three lane congestion wasn't observed during Vissim run. That happened because the capacity of the remaining two lanes was still adequate for the oncoming traffic. Although the implementation of each action-incident scenario was done at the same time and located to the same point for all the traffic situations, differences in time of the incident occurrence (congestion in three lanes) was expected.

\section{Analysis of Vissim Scenarios Towards Automatic Incident Detection}

Each incident scenario of the nine in total was applied in the microscopic simulation Vissim for every traffic situation (low-normal-high). After the Vissim run of the 27 scenarios, results for the travel times between the following Bluetooth detector sections were derived: 504-506, 504-507, 504-508 and 504-510. The travel times, produced by Vissim, were processed and converted appropriately for the TUM algorithm. In the next step the findings of the TUM algorithm were analyzed towards the performance measures DR, FAR and MTTD.

In our case the determination of DR was based only on incidents that caused congestion in three lanes. The calculation of MTTD was based on the time, required for a congestion to be developed in the Vissim run and not on the time of incident occurrence. In the context of the paper two proposed scenarios (5 and 6) of one lane closure during off-peak hour didn't cause a congestion and vehicles kept moving in free flow. These scenarios were not taken under consideration in the final analysis.

A lower DR was demonstrated in the section of the shortest detectors' distance $(2.061 \mathrm{~km})$ under low traffic demand. This decrease in DR resulted from the incidents' locations, which occurred after the downstream detector of the section. The FARs under low demand remained $0 \%$ in all sections, as it was expected due to the limited speed variations. Under normal traffic conditions the DR remained high for the sections of the shortest distance and low for the sections of $5.061 \mathrm{~km}$ and $8.061 \mathrm{~km}$. False alarms were not triggered in all sections. Under high travel volumes the values of DR and FAR increased in the sections with shorter distance between detectors $(2.061 \mathrm{~km}, 3.245 \mathrm{~km})$, while the section of the longest detectors' distance $(8.061 \mathrm{~km})$ demonstrated the lowest DR and FAR, proving that a higher DR can be achieved in shorter distances of detectors but with more false alarms. A higher FAR is demonstrated under high traffic volumes due to the speed variations of vehicles, concerning mainly detectors at close distance.

Table 1. Performance measure DR and FAR of the examined sections for low/normal/high traffic volumes

\begin{tabular}{|l|l|l|l|l|l|l|}
\hline Bluetooth Section & $\begin{array}{l}\text { DR (\%) } \\
\text { low traffic } \\
\text { volumes }\end{array}$ & $\begin{array}{l}\text { FAR (\%) } \\
\text { low traffic } \\
\text { volumes }\end{array}$ & $\begin{array}{l}\text { DR (\%) } \\
\text { normal } \\
\text { traffic } \\
\text { volumes }\end{array}$ & $\begin{array}{l}\text { FAR (\%) } \\
\text { normal traffic } \\
\text { volumes }\end{array}$ & $\begin{array}{l}\text { DR (\%) high } \\
\text { traffic } \\
\text { volumes }\end{array}$ & $\begin{array}{l}\text { FAR (\%) } \\
\text { high traffic } \\
\text { volumes }\end{array}$ \\
\hline $504506(2.061 \mathrm{~km})$ & $67 \%$ & $0 \%$ & $100 \%$ & $0 \%$ & $100 \%$ & $31 \%$ \\
\hline $504507(3.245 \mathrm{~km})$ & $100 \%$ & $0 \%$ & $100 \%$ & $0 \%$ & $100 \%$ & $7 \%$ \\
\hline $504508(5.061 \mathrm{~km})$ & $83 \%$ & $0 \%$ & $83 \%$ & $0 \%$ & $86 \%$ & $57 \%$ \\
\hline $504510(8.061 \mathrm{~km})$ & $67 \%$ & $0 \%$ & $67 \%$ & $0 \%$ & $0 \%$ \\
\hline
\end{tabular}

According to performance measure MTTD for low traffic demand the highest time to detect was recorded in section 504-507 (26 minutes) for incident 8, which is located outside the section 504-507. Moreover it is seen that, when the incident occurred at a point closer to the downstream detector station, MTTD tended to decrease.

Under normal traffic volumes the worst time to detection was recorded for the section 504-506 and more specifically for the incidents 7 and 8 . Such a great delay was expected due to the location of both incidents, which occurred outside the section 504-506. Incident 8 wasn't included in the section 504-507 also, so a detection delay of 30 minutes was recorded. This delay was almost the half of the 1 hour delay 
in section 504-506. That happened because incident 8 occurred $2.3 \mathrm{~km}$ after the sensor 506 and $1.116 \mathrm{~km}$ after sensor 507.

According to the results, on the one hand, a severe incident with two lanes blockage (incident 9) at the beginning of the analyzed section $(379+500 \mathrm{~km})$ was detected in time in all sections under high demand. On the other hand, severe incidents ( 7 and 8), with two lane blockage located after the sensor 506 and closer to the detectors 508 and 510, delayed to be detected at the first section (504-506). An incident caused by a lane closure (incident 4), $600 \mathrm{~m}$ away from detector 506 was detected in time in section 504-506. The same incident was recorded 11 and 18 minutes later in sections 504-507 and 504508, while in section 504-510 the incident wasn't detected at all. In general, the section 504-506 performed better in terms of time to detection showing a total MTTD of 6 minutes, while in the section 504-507 the total time to detection was 8 minutes.

Table 2. Performance measure MTTD of the examined sections for low/normal/high traffic volumes

\begin{tabular}{|l|l|l|l|l|}
\hline Incident Scenario & $\begin{array}{l}\text { MTTD } \\
\mathbf{5 0 4 - 5 0 6} \\
\text { low/normal/high } \\
\text { traffic volumes } \\
\text { (h:mm) }\end{array}$ & $\begin{array}{l}\text { MTTD } \\
\mathbf{5 0 4 - 5 0 7} \\
\text { low/normal/high } \\
\text { traffic volumes } \\
\text { (h:mm) }\end{array}$ & $\begin{array}{l}\text { MTTD } \\
\mathbf{5 0 4 - 5 0 8} \\
\text { low/normal/high } \\
\text { traffic volumes } \\
\text { (h:mm) }\end{array}$ & $\begin{array}{l}\text { MTTD } \\
\mathbf{5 0 4 - 5 1 0} \\
\text { low/normal/high } \\
\text { traffic volumes } \\
\text { (h:mm) }\end{array}$ \\
\hline Incident 1 & $-/-/ 0: 00$ & $-/-/ 0: 26$ & $-/-/-$ & $-/-/-$ \\
\hline Incident 2 & $0: 00 / 0: 00 / 0: 05$ & $0: 00 / 0: 00 / 0: 04$ & $0: 03 / 0: 11 / 0: 04$ & $0: 07 / 0: 11 / 0: 11$ \\
\hline Incident 3 & $0: 00 / 0: 01 / 0: 01$ & $0: 00 / 0: 00 / 0: 01$ & $-/-/ 0: 01$ & $-/-/-$ \\
\hline Incident 4 & $0: 00 / 0: 02 / 0: 01$ & $0: 03 / 0: 01 / 0: 11$ & $0: 13 / 0: 07 / 0: 18$ & $-/-/-$ \\
\hline Incident 5 & $-/ /-$ & $-/-/-$ & $-/-/-$ & $-/-/-$ \\
\hline Incident 6 & $-/-/-$ & $-/-/-$ & $-/-/-$ & $-/-/-$ \\
\hline Incident 7 & $-/ 0: 40 / 0: 11$ & $0: 00 / 0: 06 / 0: 01$ & $0: 00 / 0: 12 / 0: 03$ & $0: 02 / 0: 12 / 0: 03$ \\
\hline Incident 8 & $-/ 1: 01 / 0: 25$ & $0: 26 / 0: 30 / 0: 12$ & $0: 01 / 0: 03 / 0: 01$ & $0: 01 / 0: 07 / 0: 01$ \\
\hline Incident 9 & $0: 12 / 0: 02 / 0: 03$ & $0: 08 / 0: 02 / 0: 03$ & $0: 12 / 0: 03 / 0: 03$ & $0: 11 / 0: 09 / 0: 03$ \\
\hline
\end{tabular}

\section{Comparison of AID Based on Travel Time Data and Local Detector Data}

The 27 proposed scenarios were run for second time in Vissim, to generate local data for every detector position. The AID based on local data was done through the $\mathrm{VK}_{\text {Diff }}$ algorithm, which belongs to the category of point-based algorithms, following the comparative or pattern recognition approach. This algorithm detects an incident by comparing the speed and density of two consecutive local detectors. The speed-density difference is determined based on the following formula:

$V K_{\text {Diff }}=\sqrt{\left(\frac{v_{\text {free }}-v_{m 1}}{v_{\text {free }}}\right)^{2}+\left(\frac{\mathrm{D}_{1}}{D_{\max }}\right)^{2}}-\sqrt{\left(\frac{v_{\text {free }}-v_{m 2}}{v_{\text {free }}}\right)^{2}+\left(\frac{D_{2}}{D_{\max }}\right)^{2}}$

where, $v_{\text {free }}$ is the average free speed $(115-120 \mathrm{~km} / \mathrm{h}), \mathrm{D}_{\max }$ is the density at maximum traffic volume and $\mathrm{V}_{\mathrm{mi}}$ and $\mathrm{D}_{\mathrm{i}}$ are the mean speed and density at the measuring point respectively. If the mean speed at the local detector is higher than the average free speed, then $\mathrm{v}_{\text {free }}-\mathrm{V}_{\mathrm{mi}}=0$. An alarm for a three lane road is triggered when the $\mathrm{VK}_{\text {Diff }}$ exceeds 0.4 and traffic volume is higher than $800 \mathrm{veh} / \mathrm{h}$ (Busch, 1986).

The formula (4) takes into consideration the density and speed of every measuring point. Hence, the generated data in Vissim were the speed on every detector and the occupancy, which was used for the determination of density. A comparison of the performance of the two algorithms towards AID was done through the determination of DR, FAR and MTTD. The detection quality of both data sources (Bluetooth detectors and local detectors) in this case is exactly the same and didn't affect the algorithm results.

Overall, a greater performance of TUM algorithm was demonstrated under the three traffic situations. More specifically, $\mathrm{VK}_{\text {Diff }}$ algorithm detected more incidents under high demand than TUM algorithm, but triggered more false alarms. The total MTTD of both algorithms, under high traffic 
volumes, differ only one minute, at the expense of $\mathrm{VK}_{\text {Diff }}$ algorithm. Under normal traffic volumes, higher DR and lower FAR was demonstrated by TUM algorithm.

Many incidents were not detected by $\mathrm{VK}_{\text {Diff }}$ algorithm resulted in a lower DR. False alarms were triggered also under normal demand by $\mathrm{VK}_{\text {Diff }}$ algorithm. The total time to detection in both algorithms, under normal volumes was ten minutes. Under low traffic volumes both detectors had no false alarms. However, only the half incidents were detected by $\mathrm{VK}_{\text {Diff }}$ algorithm. TUM algorithm detected the incidents under low demand seven minutes earlier than $\mathrm{VK}_{\text {Diff }}$ algorithm, demonstrated a MTTD equal to 5 minutes. Further conclusions about the appropriateness of travel time or local data for incident detection, cannot be made. Both datasets that were analyzed, coming from simulated data and therefore their detection quality is exactly the same.

\section{Conclusion}

Under the different traffic situations, the highest performance was achieved in the section of $3.245 \mathrm{~km}$, where all the incidents were detected. In this section the number of false alarms under high volumes was low, while under normal and low traffic demand a FAR of $0 \%$ was achieved. An optimal detectors' distance could be from three to four kilometres. A shorter distance than three kilometres could result in high number of false alarms, while a longer one in lower DR. But still this distance (three to four kilometres) cannot assure the best performance due to the unpredictable nature of the incidents, whose type and location significantly affect the incident detection results. More specifically, the severity and duration of the incidents played a crucial role on their detection under the three traffic situations. Severe incidents resulting in a two-lane closure for one hour or more had a correspondingly dramatic change in capacity, making their detection easier and quicker. Incidents that didn't severely impact the capacity and lasted less than 30 minutes, were detected later or could not be detected at all, especially in sections with longer detector distance. In general, lower MTTD were achieved for incidents located closer to the downstream detectors section, even though detectors were not located in closer distance. Local data were also generated in the Vissim model for every detector location and used for automatic incident detection on $\mathrm{VK}_{\text {Diff }}$ algorithm and then compared with TUM algorithm results, with the last one demonstrating a better performance.

The findings of this study were derived from a specific motorway situation in Germany. In the future, more scenarios and field implementations in other sections will be analyzed.

\section{References}

1. Busch, F. (1986) Automatische Störungserkennung auf Schnellverkehrsstraßen - ein Verfahrensvergleich. Dissertation, der Universitaet Fridericiana zu Karlsruhe.

2. Listl, G., Singer, T. (2012) iRoute-The way to a Bavarian wide system for incident detection and journey time measurement. In: Proceedings of the 19th ITS World Congress, Vienna, Austria.

3. Margreiter, M. (2010) Reisezeitberechnung und Störungserkennung mit Bluetooth-Kennungen. Master's Thesis, Chair of Traffic Engineering and Control, Technische Universität München.

4. Margreiter, M., Spangler, M., Zeh, T. and Carstensen, C. (2015) Bluetooth-Measured Travel Times for Dynamic Re-Routing. In: Proceedings of the 3rd Annual International Conference ACE 2015, Volume 2, Global Science and Technology Forum, Singapore, pp. 447.

5. Schiereck, B. (2006) Improving Road and Tunnel Safety via Incident Management: Implementing a Video Image Processing System. In: Proceedings of the 3rd International Conference 'Tunnel Safety and Ventilation', Graz, 2006.

6. Wisconsin Department of Transportation. (2002) Microsimulion Guidelines, available at: http://www.wisdot.info/microsimulation/index.php?title=Model_Calibration\#The_GEH_Formula 